\title{
Elasto-plastic analysis of a multi-stage excavation in bonded geomaterials
}

\author{
Hamed Moghaddasi ${ }^{1, *}$ and Arman Khoshghalb ${ }^{1}$ \\ ${ }^{1}$ School of Civil and Environmental Engineering, UNSW Sydney, NSW 2052, Australia
}

\begin{abstract}
The deformation characteristics of braced and unbraced excavations in a dry structured soil are investigated numerically. A constitutive model based on the theory of bounding surface plasticity is used to predict the plastic deformation of the soil. A plastic cementation index is introduced in the hardening modules to capture soil degradation. In the model, the progress of the degradation is associated with the stress magnitude and the accumulated plastic deformation. The constitutive model is implemented in a finite difference code to analyse the lateral deformation of braced and unbraced excavations. The implemented model captures the strength increase of soils due to cementation, and predicts the subsequent debonding and strength degradation due to excavation. It is shown that soil cementation significantly influences the compression and tensile strengths of soils, and alters the deformation profile of the excavated face.
\end{abstract}

\section{Introduction}

The stability analysis of slopes and excavations composed of cemented soils (also referred to as structured or bonded soils) has been the subject of many recent investigations. Cemented soils exhibit markedly higher compressive and tensile strength compared to the parent soil, due to the presence of the inter-granular bonds. The prediction of deformations in such soils requires adequate evaluation of the destructuration phenomenon and its associated softening behaviour. This is particularly important in prediction of the lateral deformation in structured soils. The lateral deflections of both braced and unbraced excavations have been numerically investigated in several studies [e.g., 1-4]. In these works, the main focuses were to explore the capability of various constitutive models in predicting the magnitude and modes of the deformation of the excavated area. However, these studies were only concerned with predicting the deformation in soils with no bonding. For structured soils, appropriate elasto-plastic constitutive models should be employed to account for the degradation of the bonds as the excavation proceeds. Several such models have been so far proposed in the literature [e.g., 5-8]. In these models, the influence of inter-granular bonds have been considered in the size of the yield surface and in the dilatancy law, while either a strain-based hardening law [7, 8] or a stress-based hardening modulus [6] were introduced to quantify the softening behaviour due to the degradation of the intergranular bonds. In fact, most models proposed for bonded geomaterials utilize conventional plasticity formulations and, therefore, cannot predict bond destruction at small stresses when the stress point is inside the yield surface. The kinematic hardening models $[9,10]$ or bounding surface plasticity models [11-13] can overcome this deficiency. To date, several constitutive models based on these concepts have been developed for simulation of bonded geomaterials [14-16], however, the performance of these models in solving boundary value problems, such as slope stability or excavation problems, has not been fully studied in the literature.

The purpose of the presented study is to simulate supported and unsupported excavations constructed in a dry structured soil using an advanced elasto-plastic model developed in the framework of the bounding surface plasticity. The model extends the conventional bounding surface plasticity formulations to capture the effect of inter-particle bonds and the bond destruction process. The bounding surface and the plastic potentials are enlarged to reflect the effect of the bonds, and they shrink due the accumulation of the plastic work to simulate the debonding process. The constitutive model is implemented in a finite difference code (FLAC2D), and used for the simulation of the excavations. The deformation magnitude, direction and bond destruction rate are numerically determined for excavations and discussed for a range of materials with different compression and tensile strengths.

\section{The bounding surface plasticity formulation}

The mathematical formulations presented here are based on the theory of bounding surface plasticity, incorporating the effects of initial structure of the bonded geomaterials. In the bonding surface plasticity approach, the elastoplastic behaviour occurs when the stress state lies on or within the bounding surface. The distance of the stress point $\boldsymbol{\sigma}^{\prime}$ from an image point $\overline{\boldsymbol{\sigma}}^{\prime}$ on the bounding surface

\footnotetext{
* Corresponding author: h.moghaddasi.k@unsw.edu.au
} 
can quantify the hardening modules. For structured soils, the mathematical representation of the bounding surface depends on the cementation degree as well, to account for the effect of inter-granular bonds as (see Figure 1)

$$
F\left(\bar{p}^{\prime *}, \bar{q}, \bar{\theta}, \bar{p}_{c}^{\prime}\right)=\left(\frac{\bar{q}}{M_{c S}(\bar{\theta}) \bar{p}^{\prime *}}\right)^{N}-\frac{\ln \left(\frac{\bar{p}_{c}^{\prime}}{\bar{p}^{\prime *}}\right)}{\ln (R)}=0
$$

where $\bar{q}$ and $\bar{\theta}$ are the deviatoric stress and Lode angle on the bounding surface, respectively. $\bar{p}^{\prime *}$ and $\bar{p}_{c}^{\prime}$ are the modified mean effective stress and the hardening parameter on the bounding surface, respectively, defined as follows:

$$
\bar{p}^{\prime *}=\bar{p}^{\prime}+\bar{p}_{t}^{\prime}, \quad \bar{p}_{c}^{\prime}=\bar{p}_{t}^{\prime}+\bar{p}_{s}^{\prime}+\bar{p}_{m}^{\prime}
$$

where $\bar{p}_{s}^{\prime}$ is the strength variable representing the contribution of the fully debonded parent material, while $\bar{p}_{t}^{\prime}$ and $\bar{p}_{m}^{\prime}$ are the strength variables associated with the effect inter-granular bonds, as shown in Figure 1. $N$ and $R$ are two material parameters controlling the shape of the bonding surface. $M_{c S}(\bar{\theta})$ is the slope of the critical state line (CSL) in the stress space defined as [17]:

$$
M_{c S}(\bar{\theta})=M_{\max }\left[\frac{2 \alpha^{4}}{1+\alpha^{4}-\left(1-\alpha^{4}\right) \sin (3 \bar{\theta})}\right]^{1 / 4}
$$

in which $\alpha={ }^{M_{\max }} / M_{\min }$ with $M_{\max }$ and $M_{\min }$ being the slope of the CSL in triaxial compression and extension, respectively. The slope of the CSL in extension is linked to its corresponding slope in compression through $M_{\min }=3 M_{\max } /\left(M_{\max }+3\right)$. The model also requires definition of a loading surface specifying the location of the current stress state. It is assumed that the bounding surface and loading surface are homologous about a centre of homology and therefore an equations similar to that of the bounding surface is adopted for the loading surface. The direction of the plastic strain can be determined by a dilatancy law and its associated plastic potential. A plastic potential surface based on the extended Row dilatancy law is selected in this study, which includes the effect of tensile strength, as follows:

$$
\left\{\begin{array}{c}
\text { for } \mathrm{A}=0: g\left(p^{\prime *}, q, \theta, p_{0}^{\prime}\right)=q+M_{c S}(\theta) p^{\prime *} \ln \left(p^{p^{*}} / p_{0}^{\prime}\right) \\
\text { for } \mathrm{A} \neq 0: g\left(p^{\prime *}, q, \theta, p_{0}^{\prime}\right)=q+\frac{A M_{c S}(\theta) p^{\prime *}}{A-1}\left(\left(\frac{p^{\prime *}}{p_{0}^{\prime}}\right)^{A-1}-1\right)
\end{array}\right.
$$

Where $p^{*}, q$ and $\theta$ are the modified mean effective stress, deviatoric stress and Lode angle at the current stress point, respectively, and $p_{0}^{\prime}$ is a parameter controlling the size of the plastic potential surface. $p_{0}^{\prime}$ is a dummy parameter since only the derivate of the $\mathrm{g}$ is required in the model.

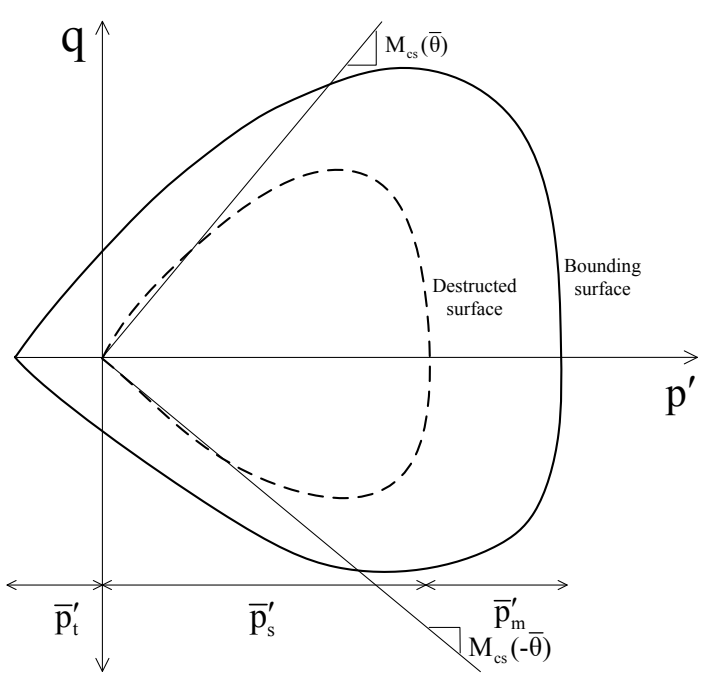

Fig. 1. The shape of the bounding surface for bonded geomaterial in stress space.

The change in the size of the bounding surface is identified via the variation of the hardening parameter $\bar{p}_{c}^{\prime}$. It is assumed that change in the plastic volumetric strain influences $\bar{p}_{s}^{\prime}$ in the standard manner, while a plastic cementation index is defined to quantify the change in the size of the bounding surface due to change in the degree of cementation through strength variables $\bar{p}_{m}^{\prime}$ and $\bar{p}_{t}^{\prime}$. To this end, the rate of the plastic cementation index is defined as follows:

$$
d I_{C}=\rho_{C}\left(1-I_{C}\right)\left(\frac{d w^{p}}{p_{1}^{\prime}}\right)
$$

Where $\rho_{C}$ is a material parameter quantifying the rate of the strength degradation of the bonded geomaterials, $p_{1}^{\prime}$ is a reference pressure, and $d w^{p}$ is the increment of the plastic work expressed as:

$$
d w^{p}=\left|p^{\prime} \Delta \varepsilon_{p}^{p}\right|+\left|q \Delta \varepsilon_{q}^{p}\right|
$$

where $\varepsilon_{p}^{p}$ is the plastic volumetric strain and $\varepsilon_{q}^{p}$ is the plastic deviatoric strain. The relations between the hardening parameter with respect to $I_{C}$ and $\varepsilon_{p}^{p}$ are taken as

$$
\frac{\partial \bar{p}_{m}^{\prime}}{\partial I_{c}}=\frac{-\bar{p}_{m}^{\prime}}{\left(1-I_{c}\right)}, \frac{\partial \bar{p}_{t}^{\prime}}{\partial I_{c}}=\frac{-\bar{p}_{t}^{\prime}}{\left(1-I_{c}\right)}, \frac{\partial \bar{p}_{s}^{\prime}}{\partial I_{c}}=\frac{-v \bar{p}_{s}^{\prime}}{(\lambda-\kappa)}
$$

where, $\lambda$ is the slope of the compression line in $v \sim \ln p^{\prime}$ plane for the debonded parent material, $\kappa$ is the slope of unloading-reloading line in the same plane, and $U$ is the current specific volume. From equations (5) and (7) and the consistency requirement, the hardening modules on the bounding surface can be obtained as follows:

$$
h_{b}=\bar{h}_{t}+\bar{h}_{s}+\bar{h}_{m}
$$

in which:

$$
\begin{gathered}
\bar{h}_{t}=\frac{1}{\left\|\partial F / \partial \overline{\boldsymbol{\sigma}}^{\prime}\right\|} \frac{\partial F}{\partial \bar{p}_{t}^{\prime}}\left(\frac{\rho_{c} \bar{p}_{t}^{\prime}}{p_{1}^{\prime}}\left(\left|p^{\prime} m_{p}\right|+\left|q m_{q}\right|\right)\right) \\
\bar{h}_{s}=\frac{-1}{\left\|\partial F / \partial \overline{\boldsymbol{\sigma}}^{\prime}\right\|} \frac{\partial F}{\partial \bar{p}_{s}^{\prime}}\left(\frac{v \bar{p}_{s}^{\prime}}{(\lambda-\kappa)} m_{p}\right)
\end{gathered}
$$




$$
\bar{h}_{m}=\frac{1}{\left\|\partial F / \partial \bar{\sigma}^{\prime}\right\|} \frac{\partial F}{\partial \bar{p}_{m}^{\prime}}\left(\frac{\rho_{c} \bar{p}_{m}^{\prime}}{p_{1}^{\prime}}\left(\left|p^{\prime} m_{p}\right|+\left|q m_{q}\right|\right)\right)
$$

where:

$$
m_{p}=\frac{\partial g / \partial \boldsymbol{p}^{\prime *}}{\left\|\partial g / \partial \boldsymbol{\sigma}^{\prime}\right\|}, m_{q}=\frac{\partial g / \partial \boldsymbol{q}}{\left\|\partial g / \partial \boldsymbol{\sigma}^{\prime}\right\|}
$$

The plastic hardening module comprises two components:

$$
h=h_{b}+h_{f}
$$

where $h_{f}$ is the hardening module on the current stress point and should fulfil the requirements of being zero on the bounding surface and infinity at the centre of homology. In this study, the following analytical function is adopted for $h_{f}$ :

$$
h_{f}=\frac{v \bar{p}_{s}^{\prime}}{(\lambda-\kappa)}\left[\frac{\bar{p}_{c}^{\prime}}{p_{c}^{\prime}}-1\right] k_{m}\left(\eta_{p}-\eta\right)
$$

where $\eta=q / p^{*}$ is the stress ratio and $\eta_{p}=(1-$ $\left.2\left(v-v_{c s}\right)\right) M_{c s}(\theta) \bar{p}_{c}^{\prime} / \bar{p}_{s}^{\prime}$ is the slope of peak strength line in the $q \sim p^{\prime}$ plane. $k_{m}$ is a material parameter and $v_{c s}$ is the specific volume at the critical state corresponding to the current stress state. To obtain elasto-plastic stiffness tensor, the following bulk and shear modulus are also considered:

$$
\begin{gathered}
K=\frac{v p^{\prime}}{\kappa} \\
G=\frac{3(1-2 v)}{2(1-v)} \frac{v p^{\prime}}{\kappa}
\end{gathered}
$$

Where $v$ is the Poisson's ratio.

\section{The analysis of a braced excavation on structured soils}

To demonstrate the application of the proposed model, a deep excavation problem carried out on a dry structured soil is simulated. The depth of the excavation is $7 \mathrm{~m}$ which is simulated in seven stages shown in Figure 2. The excavation is simulated without any anchors, and also with assuming two anchors at the depth of $2 \mathrm{~m}$ and $4 \mathrm{~m}$ below the ground surface, also depicted in Figure 2, with their mechanical properties provided in the Table 1.

The numerical model is built in FLAC2D assuming plane strain conditions, and the plasticity model is implemented as a UDM. The model parameters for performing the simulations are also given in the Table 1 . The horizontal deflections at eight points at the edge of the excavation are monitored along the depth of the excavation. The initial specific volume is assigned based on the assumption that the preconsolidation pressure in the whole layer for the reconstituted material is $700 \mathrm{kPa}\left(\bar{p}_{s}^{\prime}=\right.$ $700 \mathrm{kPa}$ ).

\begin{tabular}{|c|c|c|c|}
\hline \multicolumn{2}{|c|}{ Soil parameters } & \multicolumn{2}{|c|}{ Anchors' parameters } \\
\hline$\kappa$ & 0.05 & $\begin{array}{l}\text { Young's modulus } \\
\text { (GPa) }\end{array}$ & 210 \\
\hline$v$ & 0.2 & Yield strength $(\mathrm{kN})$ & 250 \\
\hline $\mathrm{N}$ & 1.44 & Cross section $\left(\mathrm{m}^{2}\right)$ & $5 e-4$ \\
\hline $\mathrm{R}$ & 2 & $\begin{array}{l}\text { Bond stiffness } \\
(\mathrm{N} / \mathrm{m} / \mathrm{m})\end{array}$ & $1.5 \mathrm{e} 10$ \\
\hline$M_{c s}$ & 1.1 & Bond strength $(\mathrm{N} / \mathrm{m})$ & $8 \mathrm{e} 5$ \\
\hline $\mathrm{A}$ & 2 & Spacing $(\mathrm{m})$ & 5 \\
\hline $\mathrm{k}_{\mathrm{m}}$ & 20 & & \\
\hline$\lambda$ & 0.2 & & \\
\hline$\Gamma$ & 3.216 & & \\
\hline
\end{tabular}

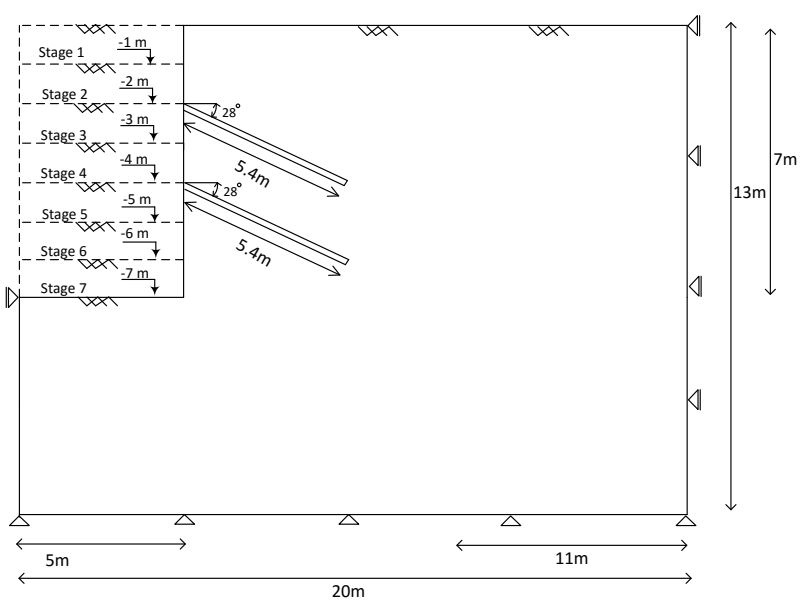

Fig. 2. The sketch of the supported excavation simulated in this study.

Table 1. The material properties used for numerical modelling of the excavation

\subsection{Effect of compressive strength due to soil cementation}

In order to investigate the effect of soil structure on the behaviour of the excavation, a set of material parameters related to the bond strength and degradation are selected. In this section, it is assumed that the soil cementation contributes only to the compression strength of the cemented soil. The parameters related to the compressive and tensile strengths due to the cementation are assumed $\bar{p}_{m}^{\prime}=0,50,100 \mathrm{kPa}, \bar{p}_{t}^{\prime}=0 \mathrm{kPa}$ and $\rho_{C}=0.1$. For the excavation without anchors, the horizontal deflection at the edge of the excavation with respect to the depth is depicted in Figure 3a for different bond parameters. It can be seen that the deformation due to the excavation is reduced noticeably by increasing the bond's strength. Another set of simulations are performed assuming the anchors. For these cases, the horizontal deformations of the excavation are shown in Figure $3 \mathrm{~b}$. As expected, the deformation due to the excavation is decreased when the anchors are in effect. Figure 3 a shows that the level of the cementation assumed and the anchors are not sufficient to prevent the large lateral displacement below the anchor levels seen in the figure. It can also be seen from Figure 
$3 \mathrm{~b}$ that increasing the bond's strength has minimum effect for the case of anchored wall. Although notable plastic deformation occurs in this case locally at the vicinity of the wall (see Figure $4 b$ ), the deformation of the excavation as a whole is basically controlled by the stiffness of the anchors. Therefore, changing the plastic parameters, such as the bond's strength parameters, has little influence on the overall response of the wall. Figure 4 shows the contour of the plastic cementation index and deformation vectors for the excavated area at the end of the excavation for the case of $\bar{p}_{m}^{\prime}=50 \mathrm{kPa}$, for excavations with and without the anchors. Since shallow excavation with material parameters corresponding to high ductility is assumed, the domain of degraded soil, which is represented by plastic cementation index of higher than zero, is propagated across a large region around the face of the excavation. Figure 4 shows that bond degradation also occurs at the base of the excavated region due to the swelling of the soil when unloading occurs. When anchors are employed, the extent of the destructuration is much smaller compared to the case with no anchors. The axial forces of the anchors are also depicted in the Figure $4 \mathrm{~b}$ at the end of the excavation.
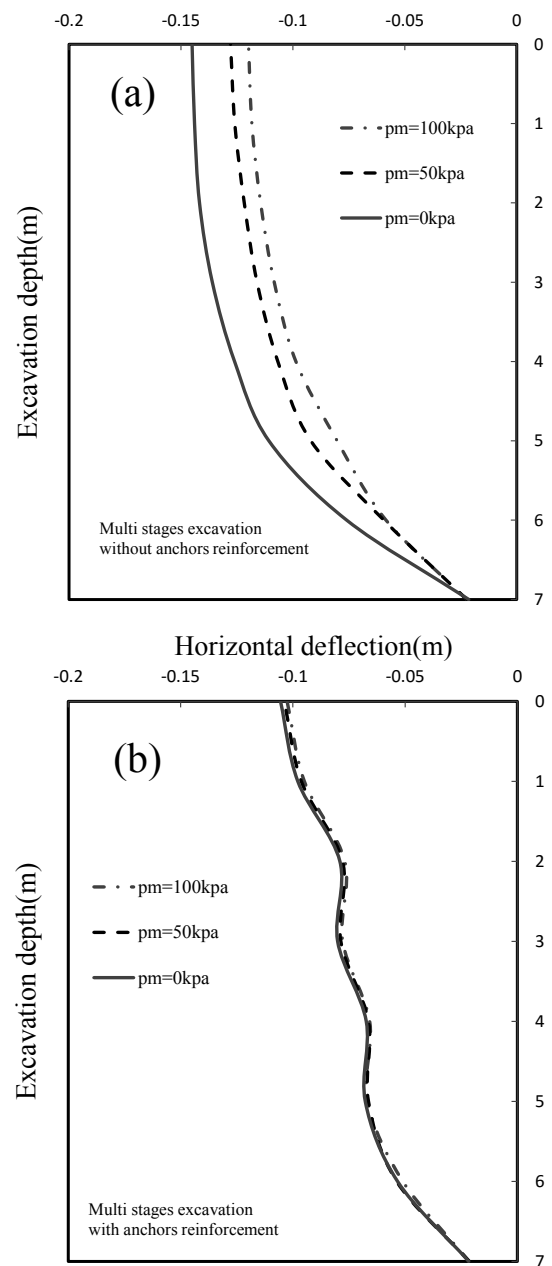

Horizontal deflection $(\mathrm{m})$

Fig. 3. Effect of bond's compression strength on the horizontal deflection of an excavation a)without anchors b)with anchors

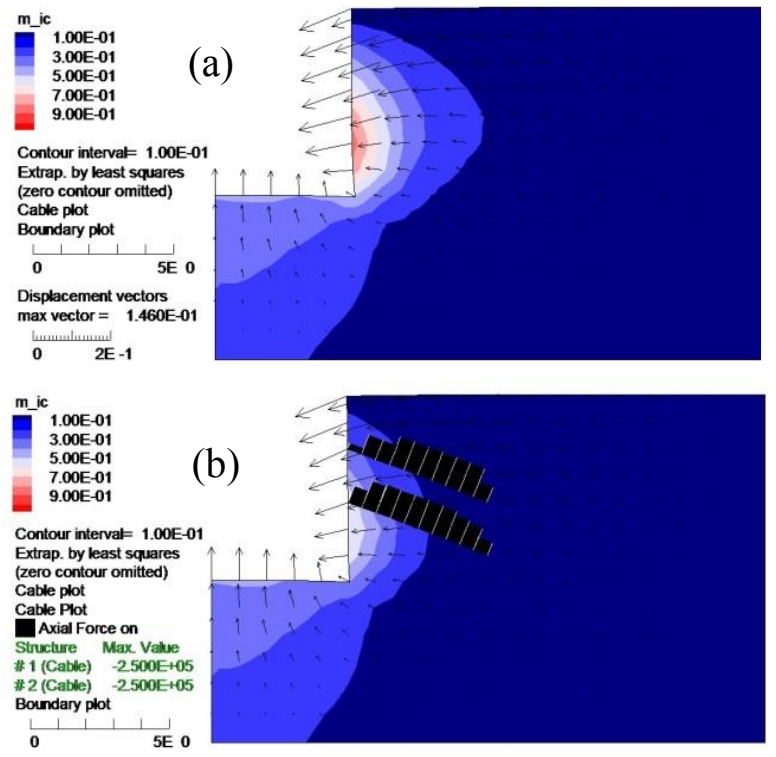

Fig. 4. The plastic cementation index and displacement vector due to excavation with $\bar{p}_{m}^{\prime}=50 \mathrm{kPa}$ a) without anchor b) with anchor

\subsection{Effect of tensile strength due to soil cementation}

The soil cementation often increases the tensile strength of the material. To explore this effect, a set of soil parameters are defined in this section to consider various tensile strength of the cemented soil, as follows: $\bar{p}_{t}^{\prime}=$ $0,50,100 \mathrm{kPa}, \bar{p}_{m}^{\prime}=0 \mathrm{kPa}$ and $\rho_{C}=0.1$. The same excavation problem is studied again using the new material parameters. The horizontal deflections of the excavation are depicted in Figure $5 \mathrm{a}$ and $5 \mathrm{~b}$ for the case of un-anchored and anchored excavations, respectively. Similar to the previous cases when the compressive strength of the soil was considered, increasing the tensile strength of the cemented soil markedly reduces the horizontal deflection due to excavation. This effect is even more pronounced compared to the cases when comparable compressive strength is considered. It is because the employment of even a small tensile strength (e.g., $\bar{p}_{t}^{\prime}=50 \mathrm{kPa}$ ) can result in elastic deformation of the excavation, and therefore small displacements. This highlights the significant effect of the tensile strength on controlling the lateral deflection of the excavation. Similar to the case of the anchored excavation with bonds' compression strength (Figure $4 \mathrm{~b}$ ), it can be noted that increasing the tensile strength of the material does not significantly change the deformation of the supported excavation (Figure 5a). The contour of the plastic cementation index and the deformation vectors are shown in the Figure $6 \mathrm{a}$ and Figure $6 \mathrm{~b}$ for an un-anchored and anchored excavation, respectively, at the end of the excavation. The regions around the base of the excavation and close to the excavation wall experiences tensile stress in excess of the tensile strength of the soil and therefore the strength degradation due to the tension is occurred in those areas. 


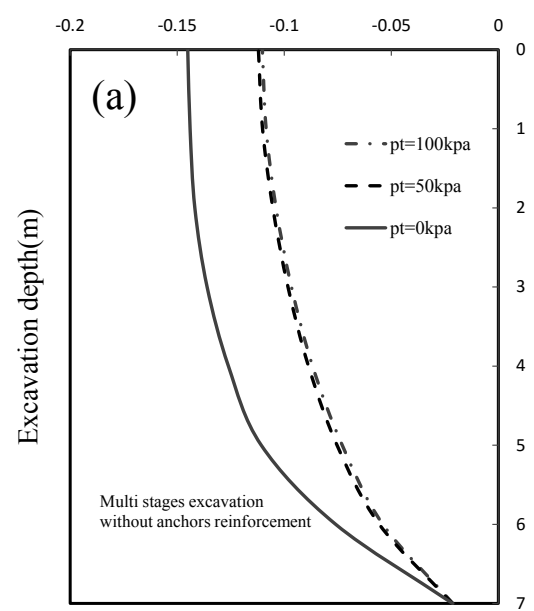

Horizontal deflection(m)

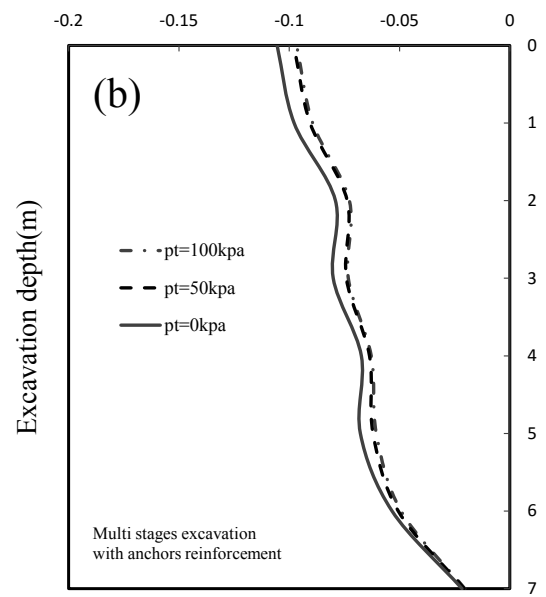

Horizontal deflection(m)

Fig. 5. Effect of bond's tensile strength on the horizontal deflection of an excavation a) without anchors b)with anchors
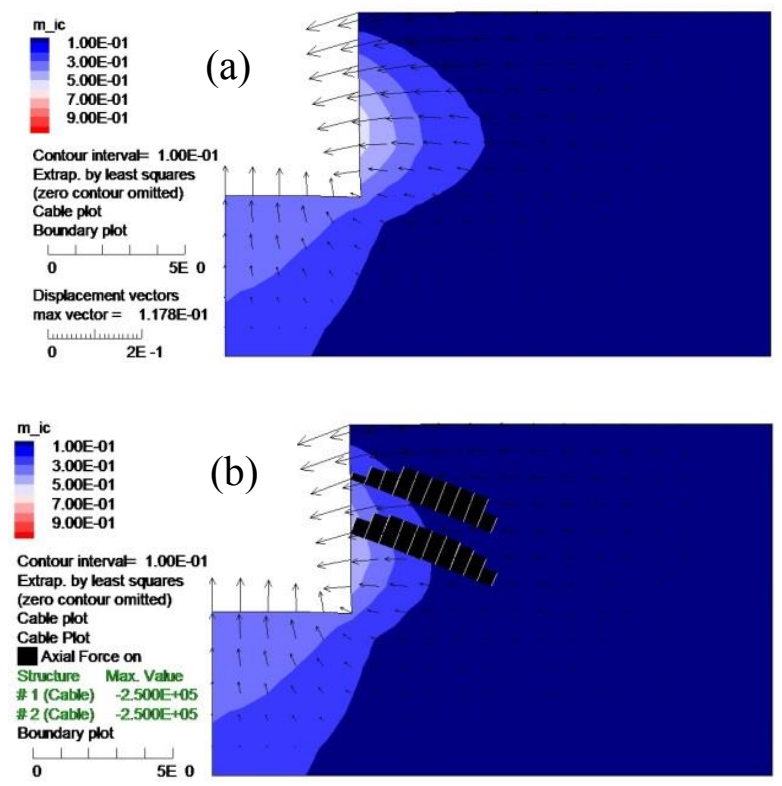

Fig. 6. The plastic cementation index and displacement vectors due to excavation with $\bar{p}_{t}^{\prime}=50 \mathrm{kPa}$ a) without anchor b) with anchor

\section{Conclusion}

The behaviour of supported and unsupported excavations in structured soils was investigated numerically in this paper. An elasto-plastic constitutive model was presented to capture the main features of the behaviour of the structured soils including strength degradation due to plastic deformations of the soil. The bounding surface plasticity framework was adopted, and the model was formulated so that it accounts for the gain in the compressive and tensile strength of the bonded geomaterials due to cementation. A work hardening approach was proposed to predict the softening behaviour of structured soils due to bond degradation. A nonassociated flow rule was adopted to capture the volume change characteristics of the behaviour. The model was adopted to obtain the lateral deflection of both braced and unbraced excavations constructed in a structured soil. Despite the low number of parameters describing the destruction process, it was shown that the model is capable of simulating the reduction in the lateral deformation of the wall due to soil cementation. Also, the use of bond's strength parameters can capture the softening behaviour of the excavation, leading to a more realistic prediction of lateral deflection. The analysis of un-braced excavation revealed that the bond strengths play a major role in curbing the wall deflection, while their effect in anchored wall are negligible since the anchors notably control the behaviour. Furthermore, the plastic cementation index of the model is an excellent index to show the extent of the damage to the initial structure of the soil, which can be used to obtain the optimum length of the anchors. The use of a single plastic index representing the destruction process is a significant advantage of the model, which facilities the design process of the anchored wall.

\section{References}

1. A.J. Whittle, Y.M. Hashash, and R.V. Whitman, J Geotech Eng-Asce. 119, 1, 69-90 (1993)

2. R.I. Borja, J Geotech Eng-Asce. 116, 6, 964-985 (1990)

3. C. Yoo, D. Lee, Comput. Geotech. 35, 2, 231-252 (2008)

4. Y.-G. Tang, G.T.-C. Kung, Comput. Geotech., 37, 6, 769-780 (2010)

5. T. Adachi, F. Oka, Int. J. Numer. Anal. Met., 19, 4, 233-247 (1995)

6. M. Liu, J. Carter, Can. Geotech. J., 39, 6, 1313-1332 (2002)

7. R. Nova, R. Castellanza, C. Tamagnini, Int. J. Numer. Anal. Met., 27, 9, 705-732 (2003)

8. W. Yan, X. Li, Géotechnique, 62, 2 (2010).

9. M. Kavvadas, A. Amorosi, Géotechnique, 50, 3, 263-273 (2000)

10. M. Rouainia, D. Muir Wood, Géotechnique, 50, 2 153-164 (2000) 
11. N. Khalili , M.A. Habte, S. Valliappan, Int. J. Numer. Meth. Eng., 63, 14, 1939-1960 (2005)

12. J. Suebsuk, S. Horpibulsuk, M.D. Liu, Comput. Geotech., 37, 7-8, 956-968 (2010)

13. Q. Chen, et al., Comput. Geotech., 61, 316-327 (2014)

14. M. Arroyo, et al., Comput. Geotech., 45, 140-150 (2012)

15. S. Mohammadi, H.A. Taiebat, Comput. Geotech., 49, 279-288 (2013)

16. S. Panayides, M. Rouainia, D. Muir Wood, Can. Geotech. J., 49, 3, 344-356 (2012)

17. M.E. Kan , H.A. Taiebat, N. Khalili, Int. J. Geomech., 14, 2, 239-253 (2014) 